\title{
Calcifying Odontogenic Cyst Associated with Impacted Mandibular Canine: A Case Report M Mardani ${ }^{1}$, L Hafezi ${ }^{2}$, N Ghadimi *3(i)
}

1- postgraduate student, Depatment of Oral and Maxillofacial Surgery, School of Dentistry, Shiraz University of Medical Sciences, Shiraz, Iran

2- Assistant Professor, Oral and Maxillofacial Radiology Dept, Faculty of Dentistry,Tehran Medical Sciences, Islamic Azad University, Tehran,Iran

3- Postgraduate student,Oral and Maxillofacial Radiology Dept, Faculty of Dentistry,Tehran Medical Sciences, Islamic Azad University, Tehran,Iran

ARTICLE INFO

Article History

Received: August 2020

Accepted: Oct 2020

ePublished:Nov 2020

Corresponding author:

N Ghadimi, Faculty of

Dentistry, Tehran Med-

ical Sciences,Islamic

Azad University,Teh-

ran,Iran

Email: omfrghadimi@

gmail.com
ABSTRACT

Background and Aim: The calcifying odontogenic cyst (COC) is a rare cystic lesion, mainly affecting the anterior aspect of the jaws. It is usually discovered in unexpected settings and can be clinically observed as a painless and well-defined lesion. The COC may be associated with other odontogenic tumors, such as odontomas. Nearly $50 \%$ of COCs are associated with an unerupted tooth, most often a canine. The most unique features of this pathology are histopathological features, including a cystic lining with characteristic ghost epithelial cells with a tendency for calcification. Radiological examinations often reveal a radiolucent and unilocular lesion, sometimes associated with radiopaque lesions. Pathological assessments are required for the final diagnosis. Management is through complete excision with annual radiographic monitoring for five years.

Case Presentation: Here, we report a classic case of COC in the left mandibular region associated with an extremely displaced impacted canine in a 16-year-old girl. Conclusion: Although uncommon, COCs are frequently associated with impacted teeth. The radiolucencies associated with impacted teeth have different effects on the surrounding structures and require different treatment plans, depending on the type of the lesion.

Keywords: Calcifying Odontogenic Cyst, Tooth, Impacted, Canine, Mandible

J Res Dent Maxillofac Sci 2020;5(4):37-41 DOI:10.29252/jrdms.5.4.37

\section{Introduction:}

The calcifying odontogenic cyst (COC), also known as "Gorlin cyst", is an uncommon developmental odontogenic lesion, which was first introduced by Gorlin in 1962. ${ }^{(1)}$ The fourth edition of the World Health Organization (WHO) Classification of Head and Neck Tumors (2017) retitled and reclassified this lesion as an odontogenic cyst; this lesion had been categorized in 2005 as an odontogenic tumor. ${ }^{(2)}$ The COC is a rare developmental odontogenic cyst. It is assessed that COC accounts for less than $1 \%$ of all odontogenic cysts of the jaws. ${ }^{(3)}$ It is associated with an impacted tooth in approximately one-third of cases. ${ }^{(4)}$ In radiographic views, COCs may be seen as a unilocular and well-defined radiolucency with or without radiopacities resembling uneven calcifications or tooth-like structures. ${ }^{(5)}$

\section{Case Presentation:}

A 16-year-old girl was referred to the maxillofacial surgery department of Shiraz University of Medical Sciences, Shiraz, Iran, with a chief complaint of an asymptomatic, non-tender swelling on the left anterior region of the mandible for one month, compatible with the area of the left lateral incisor and canine. An asymmetry was observed during the extraoral evaluation. The overlying skin appeared normal (Figure 1). The intraoral examination revealed the expansion of the mandibular anterior buccal and lingual cortical plates with a firm consistency. The swelling was non-tender. The overlying mucosa appeared normal. The third molars in all the quadrants, the maxillary second molars, and the left mandibular canine were clinically $a b$ 


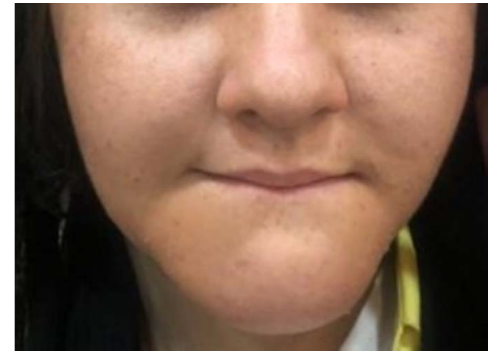

Figure 1: Facial asymmetry with normal overlying skin.

sent with severe mobility of the mandibular anterior teeth. The patient complained of a headache without any history of local trauma or abnormal conditions. The medical history was unremarkable, and the patient was not taking any specific medication.

Cone-beam computed tomography (CBCT) scans were taken to examine the lesion in detail. On the reformatted panoramic image, a large unilocular cystic radiolucency, extending from the mesial aspect of the mandibular left first molar to the mesial aspect of the right first molar, with the transverse dimension of $39 \mathrm{~mm}$ was noted with significant root displacement of all the teeth involved with the lesion. Apical root resorption was detected in right and left first premolars, central and lateral incisors, right canine, and right second premolar (Figure 2).

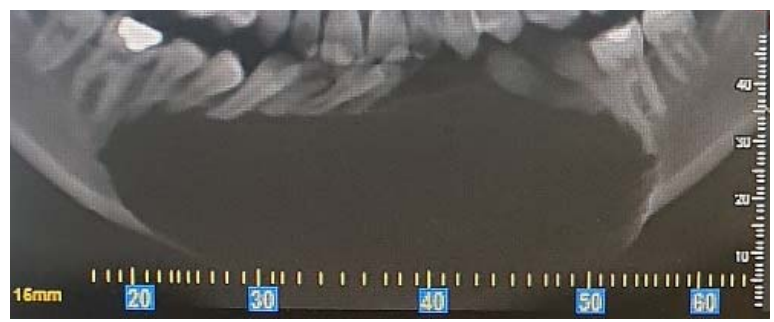

Figure 2: Cone-beam computed tomography (CBCT) scan: Reformatted panoramic image.
The axial sections demonstrated a large, well-defined, unilocular, radiolucent, and expansile lesion involving the area between the right and left first molars, measuring approximately $33 \mathrm{~mm}$ anteroposteriorly and 44.7 mm mediolaterally, with thinned buccal and lingual cortical plates and discontinuity of the buccal cortices in the area of the left lateral incisor and impacted canine (Figure 3 ).

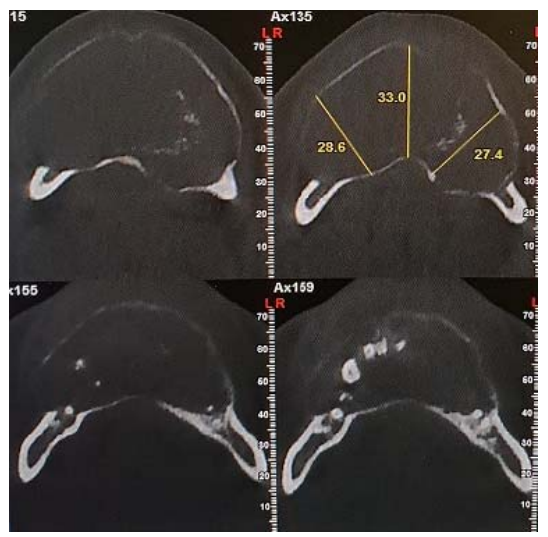

Figure 3: Cone-beam computed tomography (CBCT) scan: Axial sections.

The assessment of the three-dimensional (3D) view and the coronal sections of the lesion showed extreme displacement of the canine towards the lower border of the mandible (Figures 4 and 5).

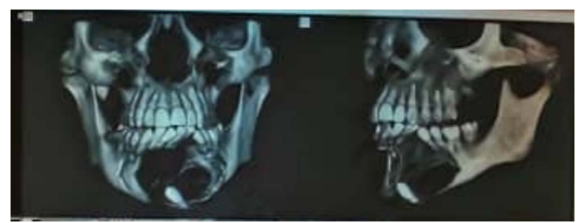

Figure 4: Cone-beam computed tomography (CBCT) scan: Three-dimensional (3D) view of the lesion showed extreme displacement of the canine towards the lower border of the mandible.

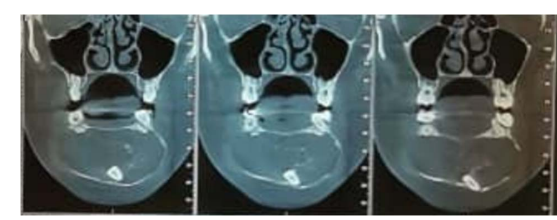

Figure 5: Cone-beam computed tomography (CBCT) scan: Coronal sections. 
The sagittal sections showed extension of the lesion with the largest dimension of $36.7 \mathrm{~mm}$ anteroposteriorly up to $34.2 \mathrm{~mm}$ superoinferiorly, containing multiple small radiopaque foci. Involvement of the inferior alveolar canal (IAC) by the lesion was detected (Figure $6)$.

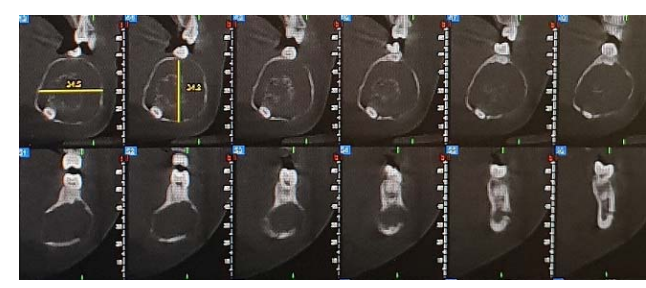

Figure 6: Cone-beam computed tomography (CBCT) scan: Sagittal sections.

The radiological differential diagnoses included COC, dentigerous cyst, ameloblastic fibro-odontoma (AFO), adenomatoid odontogenic tumor (AOT), and calcifying epithelial odontogenic tumor (CEOT).

Surgical resection was performed under general anesthesia (Figure 7).

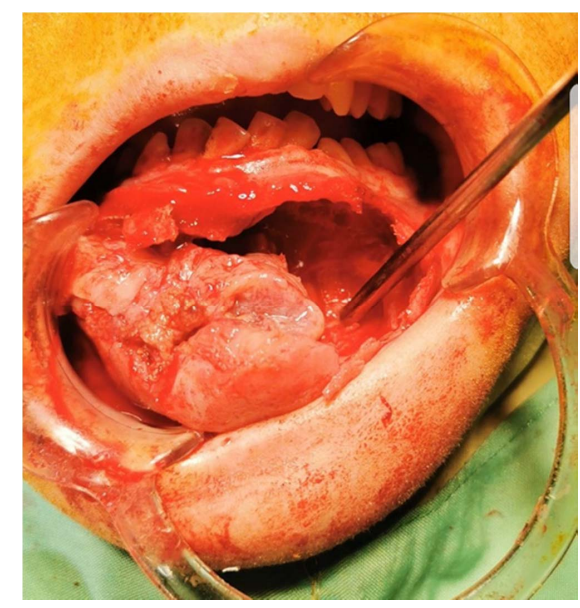

Figure7:Surgical resection of the lesion under general anesthesia.

The surgical specimen was fixed in formalin and processed. The histopathological findings revealed a lesion with an outer layer of the odontogenic epithelium of variable thickness and a basal layer of ameloblast-like cells and stellate reticulum-like cells in the suprabasal layers. Ghost cells with calcifications were also seen in the epithelium. The histopathological examination supported the diagnosis of COC (Figure 8).

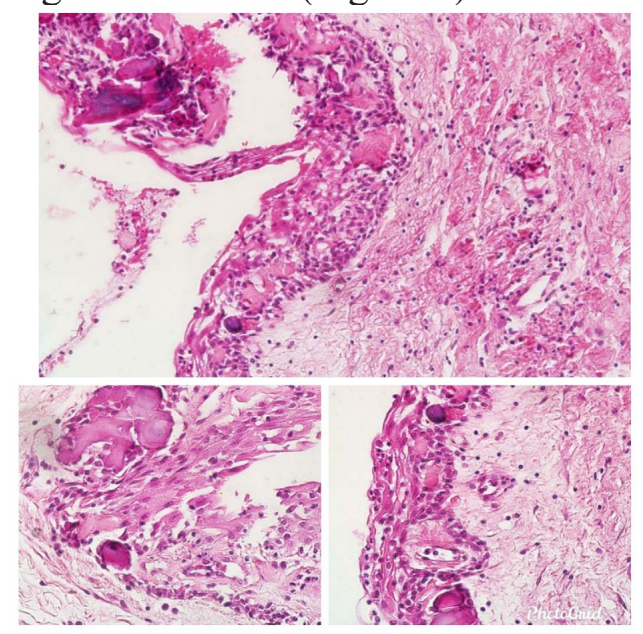

Figure 8: Histopathological sections of the lesion revealed an outer layer of the odontogenic epithelium of variable thickness and a basal layer of ameloblast-like cells and stellate reticulum-like cells in the suprabasal layers. Ghost cells with calcifications were also seen in the epithelium.

\section{Discussion:}

The COC is an uncommon lesion in the oral cavity lined by the odontogenic epithelium of variable thickness containing focal ghost cells, ${ }^{(4)}$ which most frequently occurs in the incisor and canine region, with an approximately equal frequency within the maxilla and the mandible. ${ }^{(6)}$ Asymptomatic swelling is a frequent presentation in extraosseous and intraosseous locations with an expansion of the buccal and/or lingual cortical plates. ${ }^{(7)}$ The COC is usually an incidental radiographic finding, appearing as a unilocular or multilocular well-defined radiolucency, which may contain small irregular calcified bodies associated with an odontoma or an unerupted tooth. COCs are often located in the periapical or lateral periodontal areas. ${ }^{(8)}$ Root resorption of the associated teeth has been observed in about $75-77 \%$ of the cases. $^{(6)}$ In 1991, Buchner categorized COC as peripheral and central $\mathrm{COC}$ based on 
clinical features. The COC was further subclassified into cystic and neoplastic variants. The rare malignant variant of the COC was also entered into the classification. ${ }^{(7,9)}$ The fourth edition of the WHO Classification of Head and Neck Tumors (2017) retitled and reclassified this lesion as an odontogenic cyst. ${ }^{(2)}$ COCs are generally unilocular although $5-13 \%$ of cases are multilocular. Radiopaque bodies are seen in about $50 \%$ of COCs. This lesion may have a regular outline with well-demarcated margins. Early tumors may appear completely radiolucent. With maturation, they develop calcifications and may show a mixed radiolucent-radiopaque appearance. $\left.{ }^{(10} 0\right)$ In $20 \%$ of cases, COC is associated with odontomas and ameloblastomas. ${ }^{(11)}$ The COC is a pathology that may be associated with impacted teeth. ${ }^{(12)}$ Mortazavi and Baharvand reported COC as the least common pathology associated with impacted teeth. ${ }^{(13)}$ It has also been reported that $\mathrm{COC}$ is often associated with anterior impacted teeth, ${ }^{(14)}$ similar to the location of the COC in the present case report. However, the displacement of the adjacent teeth, as observed in the present case, has been rarely reported. ${ }^{(15)}$ According to its radiographical appearance, COCs are most frequently in differential diagnosis with the dentigerous cyst, AFO, AOT, and CEOT. Considering that, in the present case report, the lesion surrounded the entire crown and root of the impacted tooth, a dentigerous cyst can be discounted. It is uncommon for COCs to appear in locations where AFOs or CEOTs commonly arise (the posterior mandible). However, this would not exclude AOTs because the incisorcanine-premolar region is the usual area involved in both jaws by AOTs and they may be associated with an impacted tooth. It may be difficult to radiographically differentiate AOTs from COCs. ${ }^{(16,17)}$ Histologically, COCs are defined as cystic proliferation with a fibrous connective tissue surrounded by the od- ontogenic epithelium. The characteristic sign for COCs is the presence of ghost cells with nuclear remnants and calcifications. Enucleation is the treatment of choice for intraosseous COCs. The extraosseous COC is treated with surgical excision. The recurrence rate is low with a good long-term prognosis. ${ }^{(3)}$

\section{Conclusion:}

Although infrequent, COCs are often associated with impacted teeth and should be included in the differential diagnosis of jaw lesions. Oral and maxillofacial radiologists and surgeons should be careful with radiolucencies associated with impacted teeth because they have different effects on the surrounding structures and require different treatment plans, depending on the type of the lesion.

\section{References:}

1. Figueiredo NR, Meena M, Dinkar AD, Khorate M. Calcifying epithelial odontogenic cyst of the mandible. J Oral Res Rev. 2014;6:57-60.

2. Wright JM, Vered M. Update from the 4th Edition of the World Health Organization Classification of Head and Neck Tumours: Odontogenic and Maxillofacial Bone Tumors. Head Neck Pathol. 2017 Mar;11(1):6877.

3. de Arruda JAA, Monteiro JLGC, Abreu LG, de Oliveira Silva LV, Schuch LF, de Noronha MS, et al. Calcifying odontogenic cyst, dentinogenic ghost cell tumor, and ghost cell odontogenic carcinoma: A systematic review. J Oral Pathol Med. 2018 Sep;47(8):721-730.

4. Neville BW, Damm DD, Allen CM, Chi AC. Oral and Maxillofacial Pathology. St Louis: Elsevier; 2016:632-690.

5. Santos HBP, de Morais EF, Moreira DGL, Neto LFA, Gomes PP, Freitas RA. Calcifying Odontogenic Cyst with Extensive Areas of Dentinoid: Uncommon Case Report and Update of Main Findings. Case Rep Pathol. 2018 May 10;2018:8323215.

6. Daniels JS. Recurrent calcifying odontogenic cyst involving the maxillary sinus. Oral Surg Oral Med Oral Pathol Oral Radiol Endod. 2004 Dec;98(6):6604.

7.Buchner A. The central (intraosseous) calcifying odontogenic cyst: an analysis of 215 cases. J Oral Maxillofac Surg. 1991 Apr;49(4):330-9.

8. Ledesma-Montes C, Gorlin RJ, Shear M, Prae Torius $\mathrm{F}$, Mosqueda-Taylor $\mathrm{A}$, Altini $\mathrm{M}$, et al. Interna- 
tional collaborative study on ghost cell odontogenic tumours: calcifying cystic odontogenic tumour, dentinogenic ghost cell tumour and ghost cell odontogenic carcinoma. J Oral Pathol Med. 2008 May;37(5):302-8. 9. Mulvihill C, Ni Mhaolcatha S, Brady P, McKenna J, Sleeman D, Fitzgibbon J. Calcifying odontogenic cyst: A case report. Oral Surg. 2020 Jan:1752-2471.

10. Phulambrikar T, Vilas Kant S, Kode M, Magar S. Cone Beam Computed Tomography Findings in Calcifying Cystic Odontogenic Tumor Associated with Odontome: A Case Report. J Dent (Shiraz). 2015 Dec;16(4):374-9.

11. Zornosa X, Müller S. Calcifying cystic odontogenic tumor. Head Neck Pathol. 2010 Dec;4(4):292-4. 12. Shiva A, Nosrati K. A Calcifying odontogenic cyst associated with complex odontoma: a case report. J Babol Univ Med Sci. 2015;17(2):57-61.

13. Mortazavi H, Baharvand M. Jaw lesions associated with impacted tooth: a radiographic diagnostic guide. Imaging Sci Dent. 2016 Sep;46(3):147-157.

14. Uzun T, Çinpolat E. Calcifying odontogenic cyst associated with the impacted third molar: a case report. Pan Afr Med J. 2019 Jun 27;33:151.

15. de Arruda JAA, Schuch LF, Abreu LG, Silva LVO, Monteiro JLG, Pinho RF, et al. A multicentre study of 268 cases of calcifying odontogenic cysts and a literature review. Oral Dis. 2018 Oct;24(7):1282-1293.

16. More CB, Das S, Gupta S, Bhavsar K. Mandibular adenomatoid odontogenic tumor: Radiographic and pathologic correlation. J Nat Sci Biol Med. 2013 Jul;4(2):457-62.

17. John JB, John RR. Adenomatoid odontogenic tumor associated with dentigerous cyst in posterior maxilla: A case report and review of literature. J Oral Maxillofac Pathol. 2010 Jul;14(2):59-62.

cite this paper as: Mardani M, Hafezi L, Ghadimi N. Calcifying Odontogenic Cyst Associated with Impacted Mandibular Canine:

A Case Report. J Res Dent Maxillofac Sci.2020;5(4):37-41 